\title{
Ethnologies
}

\section{Grand corps et mini-États ou l'image d'un tout ce que nous sommes en Tunisie}

\section{Habib Saïdi}

Volume 26, numéro 1, 2004

Littératie

Literacy

URI : https://id.erudit.org/iderudit/013347ar

DOI : https://doi.org/10.7202/013347ar

Aller au sommaire du numéro

Éditeur(s)

Association Canadienne d'Ethnologie et de Folklore

ISSN

1481-5974 (imprimé)

1708-0401 (numérique)

Découvrir la revue

Citer cet article

Saïdi, H. (2004). Grand corps et mini-États ou l'image d'un tout ce que nous sommes en Tunisie. Ethnologies, 26(1), 211-234.

https://doi.org/10.7202/013347ar
Résumé de l'article

Cet article traite de deux questions principales : le corps et l'État. L'auteur prend le cas de figure d'un spectacle de danse qui était produit et diffusé au cours des années 1990 en Tunisie. Intitulé " Nouba », ce spectacle fournit les appuis empiriques nécessaires pour que l'idée faisant de l'État un Grand Corps et du corps un mini-État ne se limite point à la métaphore, mais s'attache plutôt à la réalité. En effet, cette idée a permis à l'auteur d'appréhender le pays et le spectacle l'un à la lumière de l'autre, tout en portant l'analyse hors des sentiers battus. Cela dit, le fait que l'article traite de la question du corps dans une société arabo-musulmane ne l'implique pas automatiquement dans la sphère des études orientalistes ou islamologiques. Autrement dit, ce n'est pas de l'interdit que l'on discute dans ce texte, mais plutôt de l'inter-dit.
Ce document est protégé par la loi sur le droit d'auteur. L'utilisation des services d’Érudit (y compris la reproduction) est assujettie à sa politique d'utilisation que vous pouvez consulter en ligne.

https://apropos.erudit.org/fr/usagers/politique-dutilisation/ 


\section{GRAND CORPS ET MINI-ÉTATS}

ou l'image d'un tout ce que nous sommes en Tunisie

Habib Saïdi

CÉLAT, Université Laval

L'objectif implicite de cette réflexion sera d'étudier un pays et un corps en prenant tour à tour l'un pour une métaphore de l'autre. Cependant le pays comme corps et le corps comme pays ne feront pas ici les bornes d'un va-et-vient entre la géographie et la morphologie, ni entre la démographie et l'anatomie. Mais ils seront tous les deux traités en fonction de leurs connections et de leurs interférences, lesquelles seront appréhendées, en l'occurrence, à travers des expressions artistiques dites nationales ou populaires telles que la chanson et la danse. Dire, en fait, qu'une performance de ce genre est tunisienne, française, italienne ou autre, revient à reconnaître directement ou indirectement qu'il existe un corps collectif qui fait distinguer un pays ou une région, voire une société et une culture, par sa manière de s'articuler quand il se met à chanter et/ou à danser, que ce soit en présence des siens ou des autres.

L'idée d'un pays qui chante et qui danse, telle qu'elle sera développée dans cet article, implique donc ces formes d'articulations corporelles distinctives d'un peuple et d'un territoire. Elle implique, en outre, les politiques qui font que ces formes sont valorisées ou dévalorisées d'une époque à l'autre. Cela dit, il sera question ici d'un pays qui s'est mis à danser à un moment précis de son histoire plutôt que de l'évolution historique ou des caractéristiques générales de la danse dans ce pays.

À ce titre, le cas de figure précis que nous examinons ici est un mégaspectacle chantant et dansant qui a été réalisé et joué à l'échelle nationale au début des années 1990 en Tunisie. Intitulé Nouba, ce spectacle a consisté en un grand gala de chanson et de danse, présenté 
sur les scènes du théâtre romain de Carthage et qui a rassemblé des chanteurs et des danseurs traditionnels et modernes du pays. La particularité de ce spectacle, réalisé par Fadhel Jaziri ${ }^{1}$, tient à ce rassemblement d'artistes dont la plupart ${ }^{2}$ étaient marginalisés par les médias, soit qu'ils aient été considérés comme étant "populaires », donc des «non-artistes » selon les règles de l'art régnant jusqu'alors dans ce pays, soit qu'ils aient été perçus comme des "presque artistes » devant se limiter à amuser la populace et à fasciner les touristes.

Le «non» radical et le «presque » (plus ou moins modéré) ont focalisé les deux types de polémiques et de discours qui se sont déclenchés lors du lancement de ce spectacle, principalement en raison de son succès populaire. En effet, l'engouement du public pour ce spectacle a été immense, ce qui s'est traduit non seulement par la foule présente à toutes les représentations ${ }^{3}$, mais aussi par les réactions spontanées que ce même public a exprimées vis-à-vis de ces tableaux dansés et chantés. Tout le monde chantait et dansait Nouba en cet été de 1992 en Tunisie. Ces artistes marginalisés tels que Habbouba, Farzit, Bousaha, Hattab et tant d'autres sont soudain devenus des emblèmes dernier cri. Ils ont créé l'événement avec une danse qui n'était ni tout à fait traditionnelle ni entièrement moderne. Et c'est ce qui a fait que les polémiques mentionnées plus haut se soient centrées sur le sujet du corps en tant qu'identifiant culturel d'un peuple et comme " fondement existentiel» (Csordas 1990: 5-48) d'une culture en constante édification.

Les questions posées au sein de ces polémiques étaient : " ces chairs et ces os qui se sont mis à vibrer sur les scènes de Carthage étaient-ils des corps tunisiens?» «Ces musiques mixant le Salhit et le Jazz, le Mizoued $^{5}$ et la guitare, le Rboukh ${ }^{6}$ et le Rock and Roll, traduisaientelles un langage devenu commun à tout le monde dans ce pays?»

1. Artiste tunisien connu comme metteur en scène, dramaturge et acteur de théâtre et de cinéma.

2. En particulier ceux qui étaient appelés «traditionnels ».

3. Le spectacle a fait dix représentations en Tunisie et cinq en France au cours de l'année 1992.

4. Chant traditionnel tunisien.

5. Instrument de musique traditionnel.

6. Nom que l'on donne à l'ambiance des fêtes de mariages, à caractère plus ou moins orgiaque, dans certains quartiers populaires des villes tunisiennes. 
Autrement dit, s'agissait-il d'un montage artistique pur et simple, réunissant plusieurs genres de chants et de danses, ou était-il plutôt question d'un sujet plus profond, portant sur une certaine opération de fouille creusant dans la «nappe d'un passé virtuel » (Deleuze 1985: 170) afin de faire surgir des corps, fleurir des organes et arroser des membres? Comment, dans de telles circonstances, fouiller dans ces corps et comment étudier leurs vibrations, sinon leurs tremblements collectifs? Par ailleurs, comment appréhender les discours qui ont vu dans ces danses et ces chansons une certaine incorporation du regard de l'autre, du touriste, de l'orientaliste ou de l'amateur d'exotisme ? Le corps pourrait-il faire l'objet direct et/ou indirect des stratégies d'attraction touristique ou des tactiques de propagande?

Certes, nous ne prétendons pas apporter de réponses tranchantes et définitives à toutes ces questions. Notre but est plutôt de les reformuler à la lumière de certaines théories, celle de Pierre Legendre en particulier, laquelle sera mise en exergue tout au long de ce travail. Notre choix de nous concentrer sur la pensée de cet auteur français est justifié par l'intérêt qu'il accorde aux différentes manœuvres de montage mêlant le politique à l'esthétique dans toute société et qui, au fur et à mesure de l'évolution historique, font qu'un État finisse par prendre la forme d'un Grand corps et que des corps aboutissent à se manifester en mini-États. À ce titre, notre approche s'appuiera à la fois sur le politique et sur l'esthétique pour appréhender en même temps le pays et le spectacle.

\section{De quelques idées sur l'après colonialisme tunisien}

\section{La Tunisie du changement et le changement de la Tunisie}

La première moitié de ce sous-titre vient d'un slogan, voire d'une maxime-phare souvent utilisée par les médias et les hommes politiques tunisiens pour désigner la Tunisie de « l'Ėre nouvelle ». On tend à donner à cette expression, autant qu'à la seconde, « la Tunisie du changement », un sens positif chargé d'amener une distinction entre deux périodes politiques qui se sont déroulées depuis l'indépendance de la Tunisie en 1956. «La Tunisie du changement »'applique à l'époque de la présidence de Habib Bourguiba, de 1956 à 1987 ; « le changement de la Tunisie » qualifie la période actuelle, celle du président Zine Elabidine Ben Ali, de 1987 à nos jours. 
Le sens positif attribué au changement, dans ce cadre, constitue le fondement d'un discours politique essayant de se distinguer de son prédécesseur et parent idéologique qui, lui, se flattait d'être le libérateur et le fondateur d'un État et d'une société. Ces deux termes, qui se répètent constamment dans les discours de l'ex-président Bourguiba, relèvent du dispositif de louanges que l'on accordait à celui-ci et qui laissait entendre que c'était grâce à son génie que le pays était libéré et modernisé. L'évocation du changement constitue donc pour le nouveau régime un moyen de se libérer de cet ancien discours très paternaliste et écrasant. Il s'agit d'une « libération de la libération » comme a risqué de le dire l'auteure tunisienne Hélé Béji dans un ouvrage faisant écho aux écrits de Frantz Fanon sur le postcolonialisme. "L'indépendance nous a libérés d'une domination mais nous fait entrer dans une autre domination », dit-elle (1982: 17).

Cependant, se libérer d'un père libérateur ne relève point, dans ce cas, d'un acte de parricide, ni d'un état de dégénérescence. Puisqu'on ne tue pas ce père mais qu'on le change, on le remplace par soi-même. C'est ainsi que la signification du terme « changement » pour le pouvoir actuel en Tunisie hésite encore entre les deux sens stricts de la rupture et de la continuité. Trancher n'est pas chose aisée, car cela pourrait entraîner des conséquences désastreuses, non seulement sur ce pouvoir qui est issu de son prédécesseur comme on l'a constaté, mais aussi sur la société tout entière. En fait, les années 1980 étaient des années de crise en Tunisie. Outre les émeutes du pain ${ }^{7}$, des étudiants et des mouvements syndicaux, le pays se trouvait menacé par une montée intégriste sans précédent, d'autant plus forte que le président élu à vie, le "Combattant Suprême ${ }^{8}$ », vieillissait et que les ministres, conseillers, proches et parents qui constituaient son entourage se préparaient chacun à sa manière à s'emparer du pouvoir lors de son décès ou de sa destitution.

Les préparatifs à un changement prévisible dans les sphères du pouvoir trouvaient un écho dans la société. Vivant l'échec d'un discours prometteur de développement et de progrès, cette société devenait également la cible d'autres discours, parmi lesquels celui des fondamentalistes prônant le retour à un Islam radical. La rupture d'avec

7. Ce soulèvement populaire qui contestait l'augmentation des prix du pain compte parmi les grandes émeutes qu'a connues la Tunisie à l'époque de Bourguiba.

8. Parmi les titres de louanges qu'on accordait à Bourguiba. 
le modèle bourguibien laïc se préparait donc sérieusement hors des palais présidentiels, voire loin des cercles identifiés du pouvoir. C'est à partir de ce constat que l'on pourrait évoquer la seconde formulation, celle du «changement de la Tunisie ». Il s'agit, dans ce cas, d'un processus de renversement et d'effacement de tous les fondements et les caractéristiques de l'ordre social établi. Les fondamentalistes ont effectivement entamé ce processus dans la Tunisie des années 1980. Leurs tentatives s'étaient manifestées, entre autres, à travers des expressions corporelles "bio-sociales », comme par exemple le port du voile chez les femmes et l'engouement à se laisser pousser la barbe chez les hommes. La réaction du pouvoir fut de couper court à de telles manifestations corporelles, en interdisant le premier et en regardant d'un mauvais œil la deuxième.

Ce conflit autour du corps et de son paraître en Tunisie ne date pas des années 1980. Il remonte, en fait, à l'époque coloniale, au moment où les réformistes tunisiens admettaient que la libération du pays devait aller de pair avec celle de la société. Parmi ceux-ci, nous citons Tahar Haddad qui, dans son fameux livre "Notre femme dans la Charia ${ }^{9}$ et la société », paru dans les années 1930, appelait la femme tunisienne à se libérer de son voile traditionnel (à distinguer du voile religieux prôné par les fondamentalistes). Pour Haddad, le traditionalisme, qu'il soit imputé dans le corps ou marqué sur sa surface, alourdit davantage le poids d'indigénisation qui pèse sur le sort de la femme (Haddad 1997 : 56).

S'appuyant sur des arguments identitaires, Bourguiba s'opposait à cet appel, car pour ce leader, plus politicien que réformateur, les marqueurs culturels spécifiques à une société devaient demeurer intacts tant que cette société n'était pas politiquement libérée. Dans sa politique dite «d'étapes », la souveraineté devait précéder la modernité. L'implication dans une modernisation massive de la société devait, selon lui, être le champ de bataille d'un pays indépendant, autonome, debout, non agenouillé et non « plié ${ }^{10} »$. S'engageant dans ce champ, Bourguiba s'était lancé dans un processus moderniste rejetant presque tout rapport à la tradition. C'est pour cette raison qu'à la veille de son départ, les traditionalistes les plus radicaux s'apprêtaient à se venger, non de sa personne, mais de son modèle.

9. La loi islamique.

10. Parmi les termes se répétant souvent dans ses discours diffusés quotidiennement à la radio et à la télévision. 
En fait, le changement politique survenu en Tunisie le 7 novembre 1987, bien qu'il ait semblé marquer la simple passation de pouvoirs d'un président à l'autre, s'avère renvoyer du même coup à d'autres questions plus profondes quand on l'appréhende à la lumière de la situation du pays durant cette période. La «Tunisie du changement» est donc ce petit "pays-laboratoire » qui s'est trouvé, au moment où le monde entier s'apprêtait à des changements majeurs, confronté à des crises de toutes sortes, la plus difficile étant celle qui s'était traduite en termes d'interrogations identitaires. "Qui sommes-nous » ? La question qui se posait à l'époque coloniale comme moyen de lutte pour l'indépendance refaisait surface dans un pays ayant en principe réalisé cette œuvre depuis trente ans.

Les tentatives de réponses apportées par les traditionalistes ne pouvaient convaincre une société qui avait déjà amorcé des choix modernistes lesquels, dans les termes de Foucauld et de Derrida, s'étaient bien diffusés et disséminés dans ses corps et ses structures. Il reste donc à voir comment cette société a réagi à une telle question et comment l'État l'a rapidement ressaisie et reprise en main.

Parmi les réponses qui étaient produites dans ce cadre figurent celles que l'on pourrait qualifier d'intermédiaires ou de médiatrices. Il s'agit des réponses qui impliquent à la fois les réactions de la société et les manœuvres de l'État et qui sont souvent représentées dans le cas tunisien, dans les travaux des artistes et des intellectuels.

\section{L'art une affaire d'État, l'État une œuvre d'art}

Tout comme l'État, l'art en Tunisie, ou ce qui est envisagé en tant que tel, a toujours suscité l'attention des intellectuels. Bourguiba luimême rappelait souvent dans ses discours qu'il avait été comédien dans sa jeunesse et qu'il n'avait jamais déçu ses maîtres français en jouant devant eux Molière, Racine et Corneille. À ce titre, son jeu du pouvoir a beaucoup reposé sur l'art du spectacle, privilégiant même, si l'on peut dire, le spectacle plutôt que l'art. Les intellectuels tunisiens ont souvent fait de même. La plupart d'entre eux prennent l'art pour une affaire d'État et considèrent la construction de ce dernier comme une œuvre d'art. Dans le cas tunisien, cela revient, selon Camau, à un phénomène d'appropriation et de personnalisation de l'État: 
Lélite au pouvoir se considère comme le dépositaire exclusif du sens de l'État, par opposition à la masse des gouvernés. Elle s'attelle au renforcement de l'État en le monopolisant, au point de s'y identifier elle-même et de le gérer à la façon d'un patrimoine : l'État, c'est nous, avoue-t-elle implicitement » (Camau $1987:$ 74).

Cette formule de «l'État, c'est nous » fait écho à la question «qui sommes-nous ?». Il ne s'agit pas d'un constat de redondance, mais d'une certaine réponse qui fait en sorte que l'identité soit pensée par l'élite et décidée par l'État. Phénomène qui sous-entend que l'identité, tout comme l'art, est une affaire qui relève des compétences de ces deux partenaires et qu'elle ne concerne en rien le reste de la société.

Or, pour mieux cerner cette idée, il faut rappeler qu'à l'époque bourguibienne, l'art, ou ce qui était considéré comme tel, consistait notamment en des œuvres et des productions émanant effectivement d'une élite. Celle-ci est représentée par quelques poètes, romanciers, peintres, musiciens, hommes de théâtre et de cinéma.

Les autres formes d'expression artistique, celles qualifiées de «populaires », sont abandonnées au peuple. Autrement dit, elles ne sont pas considérées par l'État et son élite en tant qu'œuvres d'art, n'étant que du «folklore », le terme étant compris dans son sens péjoratif et s'appliquant notamment à la poésie, à la chanson et à la danse populaires. Mais, ce qui est paradoxal dans le cas de cette dernière, c'est que, bien qu'elle soit peu considérée, voire dévalorisée et délaissée par les autorités, elle s'avère être la forme artistique la plus promue et diffusée dans les programmes culturels du pays à l'étranger ${ }^{11}$. Elle est aussi utilisée comme attraction permanente dans les programmes touristiques des hôtels tunisiens.

En effet, quand on parlait de la Nouba avant sa première représentation, certains pensaient qu'elle devait constituer uniquement un spectacle pour les touristes. Mais sa représentation, au mois de juillet 1991, en tant qu'événement national au sein du festival de Carthage ${ }^{12}$, ainsi que son remarquable succès populaire ont fait d'elle le spectacle

11. Nous entendons par là les voyages presque réguliers de la «Troupe de la danse et des arts populaires " représentant le pays dans les semaines culturelles organisées à l'étranger, par le Ministère de la culture ou bien par celui du tourisme.

12. Le festival de Carthage se tient périodiquement pendant la saison estivale et ce, sur le site historique qui porte le nom de cette ville antique. 
le plus controversé de cette période. Fadhel Jaibi ${ }^{13}$ dit, dans une interview accordée au quotidien tunisien Arrai-Elaam (16 novembre 1991) que son collègue Jaziri rêvait, depuis qu'ils étaient ensemble dans la même troupe de théâtre, de trouver le moyen de dire à tout le monde, ou plutôt de permettre à tout le monde en Tunisie de dire : "voilà ce que nous sommes ». Il a trouvé ce moyen dans la danse, dit-il, là où le Tunisien devient capable de s'affirmer par son corps et de se prononcer sur lui-même.

Alors, la réponse à cette fameuse question des Tunisiens, citée plus haut, réside-t-elle dans la danse, voire dans la Nouba ? C'est ce qui fait l'objet de la suite de notre travail.

\section{La danse : un inter-dit de l'État}

Comme nous l'avons vu, la Nouba en tant que spectacle de chanson et de danse a été considérée comme une affaire d'État en Tunisie. Non seulement parce qu'elle a été soutenue par le gouvernement et qu'elle a provoqué une intense polémique chez les intellectuels, mais aussi en raison de ses échos qui se sont fait entendre chez les Tunisiens en termes d'interrogations sur leur identité et leur(s) corps.

La tendance à annoncer certaines couleurs du changement politique nouvellement survenu dans le pays renforce davantage notre idée de prendre ce dernier pour un corps et celui-ci pour un pays. Les textes de Pierre Legendre, qui nous éclaireront dans cette partie, montreront que cette idée ne se limite pas à la métaphore, mais qu'elle relève plutôt strictement du réel. À ce titre, nous allons suivre «les pas» de cet auteur pour appréhender le sens de la danse, et pourquoi pas la « danse du sens » dans ce qu'il appelle Grand corps et grand Texte.

\section{Ce que danser veut dire}

Émanant du corps, étant l'une de ses productions les plus manifestes, la parole ne se limite point, selon Legendre, à une simple expression vocale. Elle implique plutôt d'autres expressions, dont la danse. Ceci

13. Fadhel Jaibi est un metteur en scène de théâtre parmi les plus renommés dans le monde arabe et en Europe. Il a créé en 1974 la première troupe de théâtre privée de Tunisie (Le Théâtre Nouveau), avec un groupe d'artistes et d'intellectuels parmi lesquels se trouvait Fadhel Jaziri, l'auteur de la Nouba. 
dit, danser veut dire «parler» et parler, dans certains moments de l'expression corporelle, veut dire « danser»:

Parler veut dire : un corps se parle, s'énonce et se dénonce; cette machine ne produit pas seulement le vent de la voix (Factus vocis) ou ce geste privilégié par les techniciens scolastiques de l'écrit sous le nom de conduite (ductus), mais aussi la danse et ses substituts, fussentils refoulés de notre anthropologie traditionnelle (Legendre 1978 : 33).

En d'autres termes, la danse est une parole qui se prononce par le corps et qui fait que celui-ci est la traduction de l'indicible, c'est-à-dire de ce qui ne peut être dit, inter-dit (13-14). Or, pour que ce corps soit écouté, voire saisi, quand il «parle en dansant» ou quand il «danse en parlant », il faut faire en sorte qu'il résonne en caractères et caractéristiques lisibles. Cela dit, le corps s'écrit et se lit. Selon l'acception de Legendre, la danse est le moyen le plus propice pour déchiffrer toutes les inscriptions relatives au corps, celles qui l'ont déjà marqué et celles qui s'y dessinent lors de sa performance pour le faire re-marquer. Appartenant à un texte écrit en majuscule, à savoir un Grand Texte, les inscriptions régissant le corps et émanant de lui le rendent également créateur de signes. Elle le poussent à marcher et le tiennent à la verticale. Selon Legendre, la verticalité du corps est à la fois un signe de droiture et une « enseigne de droit» (34).

À ce titre, la diction par l'inter-diction est loin d'être réduite à un jeu de déconstruction. Elle tend plutôt à fixer davantage le discours sur les caractéristiques les plus saillantes du corps humain, tout en faisant de lui l'objet par lequel il devient possible d'appréhender l'« animal parlant $»^{14}$. Cela nous incite encore à dire que le corps est à la fois un outil d'écriture et une matière propice à la lecture. Ce qui s'écrit par le corps et ce qui se lit par son biais, c'est un grand Texte. Mais pourquoi ce Texte est-il grand? Parce qu'il comprend, d'après l'acception de Legendre, tous les autres textes, moins grands ou plus petits, dont ceux de la loi, de la tradition et de l'art. Le corps fait la loi parce qu'il est fait par la loi, nous signifie Legendre.

Qui plus est, le rapport corps/loi relève, entre autres, de la grandeur du Texte, de sa diversité et de sa capacité de marier le fictif au réel. Pour être plus précis, nous nous référons à la définition de ce Texte telle qu'elle est conçue par cet auteur :

14. Signifiant l'Homme, cette expression revient souvent dans les textes de Legendre. 
Fréquemment, mes travaux utilisent ce concept. J'entends par là, dans un système d'organisation, l'ensemble du dispositif de textes, socialement et historiquement repérable, qui peut-être considéré à la fois comme support et comme effet d'une reproduction ayant pour enjeu les manœuvres esthétiques et savantes de la Loi, c'est-à-dire en définitive la reproduction même de l'espèce humaine à travers une espèce culturelle particulière (2001: 41).

Ce qui serait pertinent à ce niveau, c'est de s'arrêter à cette idée où l'auteur associe l'esthétique à la loi, mais cela ne serait pas tout à fait approprié, puisqu'elle émane, tout comme la notion du Texte, d'une constante théorique qui permet à Legendre d'avancer dans ses thèses sur le corps en rapport avec la culture qui le reproduit et en fonction de celle que lui-même ne cesse de fabriquer. Par contre, cette même idée nous permet de mieux percevoir la méthode employée par cet auteur pour appréhender la danse en tant que formulations d'esthètes, après avoir été l'affaire des légistes. En disparaissant, ceux-ci ont laissé la place aux artistes et à tant d'autres, scientifiques ou théoriciens. En établissant des règles ou des canons, les uns et les autres continuent à reformuler le corps à travers des statuts qui finissent par le mettre en «statures» (Legendre $1978:$ 55).

Dans cet ordre d'idées, faire danser le corps revient à le faire marcher autrement. En d'autres termes, si la verticalité du corps humain, le fait de l'ériger en statu(r)e, est un signe de conformité à la Loi, sa « mise en danse " serait une manière de le rendre conforme aux canons de la beauté. Ceux-ci permettent à l'homme de se faire de son corps un tremplin afin de devenir un autre par et/ou pour lui-même. Il s'agit, dans la perspective de Legendre, d'une incarnation du corps idéal de l'autre se réalisant dans une optique narcissique: "une telle problématique est au cœur de la danse, institution qui fondamentalement se propose pour représenter et donner la métaphore d'un corps pour l'autre»(46).

Le corps idéal, en ce sens, constitue le revers du corps légal. En se composant et se décomposant, l'un et l'autre permettent à l'homme de se voir non seulement autrement, mais comme un autre. D'où la théorie très pertinente de Legendre, inspirée du mythe de Narcisse et portant sur «l'autre de soi », c'est-à-dire sur l'image que se fait le sujet de luimême quand il se trouve face à un miroir lui permettant de dire : "Je suis celui-là »(1994: 78). C'est ainsi que Narcisse se reconnaissait en tant qu'autre de soi. Son image reflétée sur la surface de l'eau préfigure en quelque sorte le «Je est un autre » de Rimbaud. En ce sens, le sujet 
se réfère à son altérité pour se reconnaître. Il s'identifie en termes de relation à un autre qui lui paraît être identique et en relation avec lui. Il s'agit donc d'un soi qui, sans se diviser, se réalise par l'effet de la fiction, hors du même, voire loin de l'identique. Dépassant le seuil du dicible et de l'exprimable, la danse permet la réalisation d'une telle situation, puisqu'elle sert, en l'occurrence, de miroir par lequel il devient possible d'identifier un corps en état de sur-érection sinon de surrection, un corps qui, tout en se tenant debout, s'éclate de tous les bouts.

Étudiant le corps de l'acteur qui ne diffère point, en ce sens, de celui du danseur, Eugéno Barba parle d'un désir céleste dominant l'acteur et l'attachant à un amour éternel de l'au-delà. Ainsi, pour se réaliser en tant qu'esthète et ascète, l'acteur de Barba est tenu à se tenir toujours sur la pointe des pieds, à regarder au-delà d'un mur qui s'élève en hauteur à chaque fois que cet acteur s'apprête à le dépasser (Barba 2001 : 21$60)$.

Dansant et/ou jouant, le corps incarne le ciel et la terre. Il les relie l'un à l'autre, par les bouts des doigts de ses mains et de ses pieds. Mais il les relie également par ses mythes, ses rites et ses codes, lesquels le transforment en matière à la fois source et objet d'imagination. C'est ce que Legendre appelle «la mise du corps-à-l'âme» (2001: 197). Cécile Léonardi qui, à son tour, se sert de la perspective de Legendre pour appréhender le corps et la danse, observe que «le Texte dans cette perspective serait un imaginaire somatique dominant; une version incontournable de l'imagination du corps propre à une société donnée, cette version toute orthodoxe du corps légal » (Léonardi 2000: 5372).

Dire qu'il y a un corps propre à chaque société, ou qu'une société donnée reproduit à sa manière cette matière à la fois idéale et légale, c'est dire aussi que toute société dispose d'un Texte particulier en fonction duquel elle parfait ses corps, s'annonce et s'énonce en corps parfaits. C'est ainsi qu'elle devient lisible et visible, qu'elle se transforme en sur-face, voire en territoire dont les frontières se retracent aux bouts des corps et dont le décor, la couleur et les traits de sa mise en relief, pour ne pas dire en spectacle, se font en chair et en os :

Une société fabrique nécessairement son Texte, parcelles signifiantes refoulées dans ce qui s'appelle tradition, ou encore célébrées en idéologie : inventions des normalisations en tout genres, publicitaires notamment (...) Les corps deviennent l'emblème du système où se constituent les croyances (Legendre 1978: 64). 
Certains termes introduits par l'auteur à la fin de cette citation méritent d'être soulignés. Il s'agit notamment de ce qu'il appelle emblème, système et croyances. L'usage de ces trois expressions dans son discours renvoie à des sens variés qui sous-tendent dans la plupart des cas l'articulation Corps/État. Voyons maintenant ce qu'il en ressort.

\section{La bouche, les mains et les pieds de la patrie}

Nous avançons toujours dans la perspective de Legendre. Et les métaphores que nous employons relèvent souvent de la manière particulière à cet auteur de faire le transfert entre le corps d'un côté, la société, le pouvoir, l'institution et l'État de l'autre. Il sera long de passer en revue toutes les interprétations faites par Legendre de chacune de ces expressions, mais il sera opportun de les réunir, comme il le fait d'ailleurs lui-même, autour de ce qu'il nomme le système. La marge de manœuvre qui permet de transférer les sens, c'est-à-dire de les transporter ou de les faire circuler dans la sphère que nous venons d'évoquer, devient réduite quand ce système est représenté par l'État. Car celui-ci s'incarne non seulement dans les corps par la Loi et plus amplement par le Texte, mais il se constitue lui-même sous forme de Grand Corps qui marche, parle et danse.

En d'autres termes, le recours à la métaphore devient dès lors inutile puisqu'il ne s'agit plus de transférer les sens entre un concept et un autre, mais plutôt de les voir surgir au même moment, à partir des deux concepts. Que le corps incarne l'État ou que celui-ci se manifeste en chair et en os, cela revient au même pour Legendre. Car le corps, tout comme l'État, n'est rien d'autre qu'un mot. "Nous habitons non ce que nous appelons le corps, mais un mot. Corps est un mot extraordinaire» (2001: 194). Nous pouvons dire la même chose de l'État, en partant du fait que nous l'habitons et qu'il nous habite.

Ce qui est évident pour l'auteur, c'est que l'État parle ou que, du moins, il fait comme si. La preuve c'est qu'il dispose d'une bouche, et la preuve encore c'est que sa voix se diffuse partout où ses emblèmes, voire ses images (qui ne sont autres que les corps de ses sujets), marchent, chantent et dansent. L'expression « comme si ou la verita falsas » (1994: 15), souvent soulignée par l'auteur, nous renseigne à la fois sur les moyens que ce système utilise pour parler et sur le contenu de son discours au moment où il le prononce. En fait, l'État pour Legendre affiche sa stature en se mettant debout sur des statuts institutionnalisés. Ceux-ci s'appuient 
à leur tour sur un fond théâtral, lequel permet à l'État d'avoir l'avantage de posséder la Vérité et de la dire à sa guise $(1988$ : 54 ; 2001 : 23). «La Vérité », avec une majuscule, nous renvoie au discours dogmatique de l'État. Que ce dernier se serve du dogme ou qu'il soit dogmatique ne change en rien le fait qu'il représente une création moderne des sociétés dites développées. Le dogmatisme, en l'occurrence, renvoie à un autre sens, soit « à ce qui paraît, qui apparaît, qui semble et se fait voir, jusque dans la feinte»(2001: 36).

Cette définition du dogme, qui fait écho à la «théorie de la séduction" de Baudrillard (1979) dans la mesure où celle-ci tire sa force du pouvoir des interstices, nous révèle la face la plus attrayante de l'État. Il s'agit, en fait, de la grandeur de ce corps telle qu'elle se présente et se représente. En d'autres termes, il est question de politiques étatiques non reconnues en tant que telles mais vécues et appliquées sous la forme «d'objets politiques non identifiées », des OPNI, comme les nomme Denis Martin (2002:11-45). Il s'agit en fait de la capacité qu'a ce Grand Corps non seulement de se tenir debout, mais aussi de se monter et se démonter à chaque fois qu'il se trouve en phase d'incarnation, voire de résurrection, à travers ses petits corps, pour ne pas dire ses petits sujets. En fait, «si Grand Corps il y a, il s'agit du montage légal, du corpus au sens juridique, représentable métaphoriquement par un Sujet géant qui est l'État » (Legendre 2001 : 29).

Certes cela renforce la thèse des juristes d'envisager l'État sous l'angle du droit, mais cela nous rassure également sur la pertinence de l'appréhension de Legendre quant à cette notion. Car si le droit fonde l'État, celui-ci n'est en mesure de prendre forme qu'en empruntant aux corps qu'il supervise, les mains, les pieds et tous les membres avec lesquels ils agissent pour lui donner vie et pour se faire leur propre vie. L'art rend cet emprunt plus visible, mais surtout plus subtil. Il lui permet de se réaliser à travers les interstices du comme si, soit suivant des jeux et des mises en scène où l'on peut apercevoir le Grand Corps et les petits, à savoir les mini-États $(1994: 107)$ en phase de symbiose totale. C'est ainsi que l'État, du moment où il prend le nom de patrie, voit ses droits s'élargir et son corps s'agrandir. Il atteint à ce stade le summum de sa mise en dogme puisqu'il redevient un mot. Le corps c'est un mot et la patrie c'est une fiction, rappelle encore une fois Legendre (68). 
Conformément aux postulats avancés plus haut, notre analyse de la Nouba se fera à la lumière de ces idées, ce spectacle nous servant de cas de figure à travers lequel nous pourrions concrétiser certains aspects des rapports corps/État tels qu'ils sont réfléchis par Legendre. Qui plus est, ce cas est intéressant en ce qu'il porte sur un pays où l'État ne se présente pas seulement en tant qu'instance politique, mais aussi en tant que personne morale qui se voit chargée de l'éducation d'un peuple et de l'élaboration d'une culture. Nous rappelons à cet égard la personnalité charismatique de l'ex-président Habib Bourguiba qui, comme son idole Mustafa Kemal Atatürk ${ }^{15}$, se prenait pour un père de la nation et pour un saint, comme le constate Kerrou (1998: 81-102). Nous rappelons également le fait que l'État, au moment de l'indépendance de la Tunisie, était accaparé par l'élite qui, à l'image de Bourguiba, s'identifiait à l'État et se considérait comme son incarnation. Ainsi, il ne sera plus surprenant de constater que cette question identitaire posée par les Tunisiens, à savoir «qui sommes nous?", soit aussi une préoccupation majeure de cette élite et donc une affaire très délicate de l'État.

En termes juridiques, certainement chers à Legendre, la Tunisie, ainsi qu'on le lit dans sa Constitution, s'affirme en tant qu'État arabe et musulman. Mais cette affirmation est loin de convaincre une élite qui opte plutôt pour l'élargissement de son champ d'appartenance, avançant à cela deux raisons : la première relève de l'histoire du pays qui ne peut être réduite à son islamité et à son arabité ; la deuxième tient au discours très modernisateur de cette élite. Certes, de tels aspects ne sont pas aussi clairement énoncés que l'on pourrait le croire, mais sont plutôt latents et implicites. Ils se situent au niveau de l'inter-dit et ils relèvent du dogme. En suivant la perspective de Legendre, il nous est possible de les apercevoir à travers les interstices de ce spectacle. Et pour commencer, nous allons regarder du côté du lieu où celui-ci était joué.

\section{Carthage et le premier coup du tambour}

Carthage n'est pas seulement un lieu de mémoire pour les Tunisiens. Elle est aussi un haut lieu de pouvoir. Le palais présidentiel, qui se trouve à proximité du théâtre antique où l'on a donné la Nouba, confère à ce site un caractère à la fois politique et historique. Ainsi peut-on

15. Fondateur de l'État turc qui succéda à la khilafa [royauté] à la veille de la chute de l'empire ottoman après la première guerre mondiale. 
comprendre pourquoi l'État, depuis les Phéniciens, s'approprie la plupart des événements qui se produisent sur ce site et pourquoi les artistes, en montant sur les scènes de son théâtre, se sentent frôler le pouvoir, sinon le détenir et le prendre. Dans les termes de Legendre, jouer à Carthage c'est faire comme si le corps d'un pays géographiquement petit soit plus grand que son territoire. En ce sens, l'ampleur de « 3000 ans d'histoire ${ }^{16}$ et la résonance de noms puniques célèbres tels que Hannibal, Hasdrubal, Jugurtha et la reine Didon sont implicitement restitués dans l'imaginaire des Tunisiens, voire dans leurs corps, dès que le spectacle commence.

Quand Lotfi Bouchnack, un illustre chanteur tunisien connu pour sa voix mélodique et très forte, se met le premier à chanter dans ce spectacle «la gloire des Hommes de la Tunisie », celle de «tous les hommes dans leur ensemble» dit-t-il, ceux qui sont venus très nombreux sur les gradins du théâtre ne se demandent plus « qui sommes-nous? »; ils s'immobilisent plutôt en se mettant debout, comme s'ils s'attendaient à ce que quelque chose arrive. Et en effet, ce quelque chose arrive : on l'entend dans leurs cris de joie et on le voit sur leurs corps dès que le premier coup du tambour résonne. Celui-ci déclenche en eux leur Nouba collective, soit leur danse ordonnée mais non préparée. En Tunisie, on dit que chacun a sa Nouba, c'est-à-dire son rythme préféré qui lui rend la danse irrésistible. On dit aussi que la Nouba commune à tous les Tunisiens est celle qui se déclenche ainsi tout d'un coup, voire à l'improviste, et qui fait danser tout le monde au même rythme.

Mais la danse des Tunisiens à Carthage était-elle improvisée? Autrement dit, comment expliquer cette immobilisation des corps et ce silence des gestes qui se manifestaient chez ces spect-acteurs (Boal 1993 :28) avant le premier coup du tambour ? Certes, ce dernier ne représente pas une cloche pavlovienne pour les Tunisiens. Mais il peut constituer, comme pour toutes les sociétés d'ailleurs, un écho du pouvoir, voire le moment où un État devient capable de rythmer par le même coup les battements des cours et des corps de l'ensemble de ses sujets. Ainsi, il serait pertinent de le prendre pour un État maestro, qui gouverne par la baguette tout en s'immergeant dans l'orchestre afin de se faire entendre à travers les symphonies et les applaudissements de son public. Revenons encore à Legendre pour rappeler ceci :

16. C'était, d'ailleurs, le titre d'un grand spectacle tenu à l'échelle nationale et qui racontait l'histoire du pays depuis la fondation de Carthage jusqu'à l'arrivée au pouvoir du président Zine Elabidine Ben Ali. 
Un bâtiment non seulement doit tenir debout selon les lois de la physique, mais aussi doit avoir l'air de tenir debout; il a la force d'une image. Il en est ainsi de l'État : ce sont les rites et les spectacles qui le font exister pour en faire une image au regard des croyants que nous sommes. On n'a encore jamais vu, on ne verra jamais gouverner une société sans musique, sans les chants, sans les récits fondateurs, sans les drapeaux et les emblèmes. La fameuse formule de Jakob Burckhardt sur l'État comme œuvre d'art est à prendre à la lettre (1999: 55).

Il est clair que l'expression avoir l'air, soulignée par l'auteur, fait référence à celle de comme si. Elle fait écho également à la notion d'interstice. Mais ce qui la rend encore plus intéressante c'est qu'elle est employée dans ce contexte pour évoquer la force de l'image. Une force qui, sans doute, ne peut servir qu'à l'État. Comme nous l'avons indiqué plus haut, l'engouement de ce Grand Corps à dresser les autres corps et à les faire marcher à la verticale l'incite à se servir à la fois du juridique et de l'esthétique. Le plus beau serait le plus droit, le plus petit restant toujours beau, mais à condition de se faire à l'image du plus grand.

C'est ainsi que l'on peut appréhender la danse des groupes de jeunes, notamment des jeunes femmes, impliqués dans le spectacle. Ils étaient placés sur des praticables, à un niveau plus élevé que l'orchestre et les chanteurs. Habillés en costumes transparents et exposés à un jeu de lumière distinguant surtout leurs hanches, seins et cheveux, ils donnaient l'impression de constituer les miniatures variées d'un corps unique mais dispersé. Idée qui nous permet de regarder davantage à travers les fentes, non de ces costumes transparents, mais de l'État et du spectacle.

Le discours de l'État relayé par les médias en Tunisie insiste souvent sur le dynamisme (économique ou autre) du pays, sur la vivacité de sa population, l'authenticité de sa culture et la beauté de ses paysages. Pour cet État, comme pour tout autre d'ailleurs, la stratégie la plus efficace pour mettre ces qualités en valeur est certainement de les inscrire sur les corps et de les diffuser en images. Les corps circulent et les images voyagent. Ce qui permet au pays qui semble être fixé sur son territoire de se déplacer, voire de se disperser en miniatures de visages et de paysages. Certes, cela nous rappelle les idées d'Appadurai sur les « scapes » et sur « l'image, l'imaginé et l'imaginaire » (Appadurai 2001 : 66). Mais ce qui nous rattache encore à la pensée de Legendre quant à cet aspect, c'est qu'elle fournit à ce concept des fondements historiques et institutionnels. 
Pour Legendre, un territoire et une population se ressemblent par les traits respectifs de leurs visages et de leurs paysages. Ils s'unissent et se rassemblent également en fonction des images qui les distinguent et illustrent leurs références. Au regard de ce contexte, «l'État comme concentré symbolique [...] demeure le Maître des images, au sens où il conserve la garde de la Référence. Autrement dit, l'État reste en position de garantir l'image du Père, soit l'image de Dieu : l'Imago Dei» (1994: 31-32). Ceci dit, les corps dansant des jeunes tunisiens dans ce spectacle constituent les reliefs, voire la géographie, d'un territoire qui ne cesse d'être reconfiguré par l'État. Ils représentent tout au plus des images en miniatures de ce Grand Corps, des reflets de sa taille et des traces de sa marche, voire des vibrations de son rythme. En ce sens la danse, telle qu'elle a été montrée dans ce spectacle, prend la forme d'un territoire qui se fait corps. Il bouge, souffle et respire. Il se montre vivant en s'inscrivant sur des visages et en se faisant distinguer en paysages. Et l'auteur d'ajouter :

Le territoire est une traduction politique, car il est d'abord, comme disait l'art de la Renaissance, le portrait de la terre, une image monumentale, où l'homme cherche à saisir ce qu'il y a de plus humain pour l'homme, une ressemblance avec lui même. [...] Le territoire est une humanité mise en forme, qui fait corps avec les lieux, les déchiffre, les habite. [...] Les paysages sont le Miroir des populations... Notre lien à la géographie reste nostalgique, car la géographie témoigne de la plus ancienne écriture $d u$ Monde, à travers laquelle nous cherchons un monde à notre ressemblance et qui nous parle (1999: 31).

Mais, si la Nouba s'avère être une écriture d'un certain monde tunisien et une description de ses paysage, dans quelle langue a-t-on écrit et décrit ce monde ? Comment ces corps l'ont-il exprimé et interprété et comment se situent-ils par rapport à lui ? Est-ce qu'ils le précèdent et le portent à l'avance ou bien sont-ce eux-mêmes qui représentent ses produits et qui préfigurent son émergence?

Pour répondre à ces questions, nous prenons l'exemple d'un corps singularisé. Le corps de quelqu'un qui a failli accaparer à lui seul le spectacle uniquement parce qu'il s'est distingué par sa danse et, surtout, parce qu'il a fait de son tronc, ses pieds, ses mains et tout le reste de ses membres, des langues qui se parlent au même moment, voire des syllabes gestuelles qui s'articulent pour constituer en fin de compte un non-sens appelé la danse Habbouba. Nous parlons de non-sens, car cette danse, 
comme on le verra, n'est ni occidentale, ni traditionnelle, ni d'ailleurs tunisienne bien qu'elle soit devenue, depuis l'avènement de ce spectacle, l'une des danses les plus célèbres en Tunisie.

Il s'agit, en fait, d'une performance qui a été réalisée dans le cadre de la Nouba par un artiste appelé Habbouba. Âgé d'une cinquantaine d'années, connu comme chanteur et danseur traditionnel, il faisait partie, avant la production de ce spectacle, de ces artistes marginalisés par les médias et les intellectuels, bien qu'il fût très apprécié et très célèbre dans les classes populaires, notamment dans les faubourgs de Tunis. Son apparition dans le spectacle de la Nouba s'est distinguée par ses danses beaucoup plus que par ses chansons. Quand il se met à danser, Habbouba se tient nettement en position debout, tout en dressant son corps avec beaucoup d'orgueil vers le haut. Il gonfle sa poitrine, lève ses bras au ciel et nuance l'articulation de ses doigts. C'est comme s'il cherchait à indiquer qu'il n'a pas encore atteint toute sa hauteur ou comme s'il faisait glisser ses mains à travers des plis de nuages afin de monter de gigantesques escaliers menant à des extrémités célestes. Ses épaules le portent encore davantage vers le lointain en allant chacune dans un sens ou en faisant osciller sa poitrine et son buste. Ses hanches quant à elles prennent la forme circulaire de l'orchestre du théâtre antique de Carthage. Elles tournent en rond tout en se fixant sur des jambes témoignant d'une élasticité remarquable. Ses pieds enfin tapent le sol, faisant écho aux coups du tambour et aux applaudissement du public.

Si l'on essaie d'identifier ces gestes en termes de danses connues dans le monde, l'on dirait que les pieds de Habbouba dansaient le flamenco, ses jambes la samba, ses hanches le fezzani (une danse orientale répandue en Afrique du Nord), ses épaules le rock and roll, etc. Or, conformément à la perspective de Legendre qui considère la danse comme une parole, voire un langage rendant visible et lisible ce fameux Grand Texte, l'on pourrait dire que le corps de Habbouba incarnait et extériorisait plusieurs langues à la fois. Il écrit par ses mains et nous fait lire par ses pieds. Il ponctue l'espace de ses yeux tout en orientant nos regards vers des points, des virgules et des traits d'union faisant de son corps un vrai texte. Celui-ci enseigne et renseigne : il enseigne la calligraphie du geste ; il renseigne sur la multiplicité des temps et des lieux servant de socle à ce spectacle; il diversifie ses phrases et ses vocables. "Nous sommes à la croisée des chemins, rappelle Jaziri, et il 
est temps pour nous de nous prononcer en voix multiples et de nous exprimer par des métalangues $»^{17}$.

Roseau et parchemin, le corps dans la Nouba traduit des langues et transcode des écritures. Les textes qu'il a produits nous incitent à appréhender davantage la portée de ses gestes et le sens de ses articulations.

\section{En guise de conclusion : l'esthétique chair/terre}

La dualité écriture/lecture très développée au sujet du corps constitue en quelque sorte une constante théorique qui fait couler beaucoup d'encre. L'étude de ses origines dans l'histoire de l'écriture nous semble prometteuse d'une autre étude qui pourrait prolonger celleci, car l'idée d'inscription et d'impression se rapportant à la chair humaine mérite d'être amorcée. Mais, indépendamment d'un déroulement historique, il suffit de nous limiter à notre époque pour constater l'engouement des individus d'une part, des institutions d'autre part, à s'approprier des archives et à s'inscrire sur des pages réelles et/ou virtuelles. Ainsi que le démontrent de plus en plus les travaux récents en sciences humaines et sociales, le corps s'avère être la page principale où l'on écrit tout ce que nous avons mentionné (voir à titre d'exemple Csordas 1994: 12 ; Certeau 1980 : 243-244).

Qui plus est, le corps-archive donne lieu au corps-mémoire, c'està-dire à une œuvre de l'histoire qui se fait écrire par soi et par les autres. Faisant référence à Pierre Nora (1988), Paul Ricœur rappelle que le corps est le « lieu primordial de la mémoire, l'ici par rapport à quoi tous les autres lieux sont là-bas » (Ricœur 2000 : 51). Connerton fait de même. Il considère que l'incorporation va de pair avec l'inscription et que la matière sur laquelle on écrit et on s'écrit est constituée de chair et d'os. Selon cet auteur, certaines versions du passé sont préservées et transmises sous formes de représentations par des paroles et des images, mais d'autres se servent des corps pour se représenter, voire pour le représenter :

Our bodies, which in commemorations stylistically re-enact an image of the past, keep the past also in an entirely effecive form in their continuing ability to perform certain skills (Connerton 1989: 72).

17. Cette citation de Fadhel Jaziri est extraite de l'une des entrevues que nous avons réalisées avec lui à son domicile à Tunis au mois de juillet 2003. 
La mémoire, qui se mêle à la tradition et à la culture, fait que ces propos de Ricœur et de Connerton fassent écho à ceux de Legendre. Pour ce dernier, la tradition est définie comme " une répétition du Texte » (1978: 83). N'oublions pas non plus qu'il voit dans le Grand Corps un garant suprême des emblèmes, des symboles et des références quant à une société donnée. Par ailleurs, nous avons déjà signalé l'une des raisons qui ont conduit Jaziri à réaliser ce spectacle. D'après son collègue Jaibi, il s'agissait de dire " voilà ce que vous êtes " à des citoyens qui se demandaient «qui sommes-nous?». Le témoignage de Jaibi mérite d'être appréhendé à la lettre, quoi qu'il en soit de ses intentions réelles.

Ce qui importe le plus dans ce témoignage, c'est son langage déictique. La formule « voilà ou voici » sert de démonstratif, c'est-àdire d'indication réelle et pratique. "Elle pointe du doigt un certain vis-à-vis » comme le constate Barthes, précisant qu'il s'agit d'une caractéristique propre aux arts du spectacle (1980:16). Or, ce qu'il est question de montrer à travers la Nouba, ce sont les Tunisiens. Sans nier cette thèse ni la confirmer entièrement, Jaziri reconnaît avoir eu recours "au peuple pour communiquer au peuple » et pour dire ainsi à ces Tunisiens: «vous êtes dans la beauté ». Ce qui frappe l'œil dès que l'on se fie à ce propos, ce sont les corps de ces Tunisiens, écrits et d-écrits en caractères mémoriels. La présentation de la Nouba sur un site historique ainsi que ces renvois esthétiques au patrimoine et à la tradition (quoique modernisés), répondent à un discours devenu dominant au cours des années 1990 au cœur de l'État et chez les intellectuels en Tunisie. D'ailleurs, la plupart des films tunisiens qui étaient produits durant cette période ont tous révélé de tels caractères mémoriels ${ }^{18}$.

Pour nous limiter à la Nouba, nous constatons donc que ses réalisateurs avaient pour intention de se servir du site et des corps pour mettre en éclats le mémoriel. C'est comme si on cherchait à montrer du doigt, encore une fois, que la chair est tellement rattachée à la terre qu'il suffit de creuser dans l'une ou dans l'autre pour faire surgir une multitude de vestiges, d'artefacts, de gestes, de souffles, de voix et de paroles. D'où il devient opportun pour l'artiste, en ce sens, de se prendre pour un archéologue s'appropriant le corps comme chantier de fouilles et faisant du «passé la tapisserie du présent » (Béji 1994: 58).

18. Nous citons à titre d'exemple, «Halfaouine » de Férid Boughdir, «Costume » de Kalthoum Bornaz, «La danse du Feu » de Salma Baccar. 
En fait, que ces fouilles concernent le sol, le corps, le passé ou le présent, ce genre d' «obsession mémorielle » devient de plus en plus répandu chez les artistes et les intellectuels tunisiens. L'historienne Lucette Valenci se sert de ses promenades aux alentours de Carthage, où elle s'amusait à récolter des fragments de mosaïque, pour monter un texte sur la diversité sociale, culturelle et historique de la Tunisie. Le montage de ce texte se révèle presque identique à celui du spectacle de la Nouba, puisque l'on insiste dans les deux cas sur l'apparat de cette mosaïque et sur sa capacité, encore, de montrer du doigt une certaine esthétique qui fait alliance entre la chair et la terre (Valenci $2000: 24$ 29).

La terre sur laquelle on danse et qui nous fait vibrer, voyage et se déplace à son tour. Elle nous accompagne ou elle part sans nous en images et en souvenirs ensevelis dans les imaginaires des autres. Autrement dit, les fragments de mosaïques récoltés par Valenci et partis avec elle, comme le dit son texte, depuis son installation en France (24-29), pourraient être remplacés, de nos jours, par les cartes postales, les objets artisanaux et tout autre produit touristique se trouvant exposé sur le site. On pourrait encore leur substituer les échos d'un spectacle tel que la Nouba, par ses avatars qui continuent à meubler le paysage culturel tunisien, ainsi que par ses images diffusées à l'intérieur et à l'extérieur du pays dans le but de charmer les siens et les autres.

En effet, ceux qui pensaient que la Nouba était un spectacle pour les touristes ne se trompaient pas. Elle l'est effectivement. D'abord parce qu'elle était produite sur un site touristique ou, du moins, potentiellement ouvert aux touristes; ensuite parce que le sens de ce dernier terme (les touristes) ne signifie en rien qu'il ne s'applique pas aux visiteurs provenant du même pays. Au contraire, il y a plus d'une raison pour parler, de nos jours, d'endotisme comme l'on parle d'exotisme. L'admiration de soi est d'une telle importance que l'on ne peut l'ignorer ni la placer après notre désir de l'autre. Pour fasciner celui-ci, pour le séduire et pour le transformer en miroir reflétant nos belles images, nous avons besoin de l'apprécier à la lumière de nos " matériaux narcissiques » comme le dit Legendre (1994: 92).

Pour terminer, évoquons cette symbiose du voyage et de la danse telle qu'elle est appréhendée par Kracauer. Celui-ci constate que ce qui caractérise les sociétés modernes, c'est leur manière de vivre l'espace et le temps. Cela se manifeste à travers deux pratiques très distinctives 
de ces sociétés, le voyage et la danse. L'engouement pour le premier se traduit par le désir de s'orienter vers des espaces situés « nulle part dans l'ailleurs ». Le charme de la deuxième vient de ses formes, ses expressions et ses recettes presque alchimiques faisant du corps une matière infinie et indéfinie dans l'élaboration de l'être et de l'existence.

En tant qu'institutions formalisées, voyage et danse sont depuis longtemps énormément compromis. À quel pays, à quel pas de danse accorder sa préférence, ces questions sont liées sans doute, comme celle de la coiffure, aux instructions de cet étrange et a-sacral Anonyme, dont la société qui donne le ton suit aveuglément les caprices; prendre le large, dans l'espace et le temps, telle est, semblet-il, l'exigence (Kracauer 1996: 26).

"Qui sommes-nous? Legendre donne une réponse à cette question. Non seulement aux Tunisiens, mais à tout le monde semblet-il. Pour se reconnaître, "pour instituer le semblable, une société construit... l'image d'un tout ce que nous sommes" (1994: 64). À ce titre, nous pourrions dire que la Nouba tunisienne fait partie de cette image et que chaque société dispose également de sa Nouba qu'elle chante et qu'elle danse quand elle ne trouve plus rien à dire, quand elle se décide à tout inter-dire. 


\section{Références}

Appadurai, Arjun, 1995, Après le colonialisme : Les conséquences culturelles de la globalisation, Paris, Payot.

Barba, Eugeno, 2001, "L'essence du théâtre », dans Josette Féral, dir., Les chemins de l'acteur. Québec, Éditions Québec Amérique : 2160.

Barthes, Roland, 1980, La chambre claire : Notes sur la photographie. Paris, Gallimard-Seuil.

Baudrillard, Jean, 1979, De la séduction: l'horizon sacré des apparences. Paris, Galilée.

Béji, Hélé, 1982, Désenchantement national : Essai sur la décolonisation. Paris, François Maspero.

_ 1994, L'art contre la culture : Nûba. Paris : Intersignes/Essai.

Boal, Augusto, 1980, Stop c'est magique. Paris, Hachette.

Bourdieu, Pierre, 1980, Le sens pratique. Paris: Les Éditions de Minuit. Camau, Michel, 1987, «État, espace public et développement: le cas tunisien », Annuaire de l'Afrique du Nord, XXVI : 67-78.

Certeau, Michel de, 1980, L'invention du quotidien: Vol. 1/Art de faire. Paris, Union générale des éditeurs.

Connerton, Paul, 1989, How Societies Remember. London, Cambridge University Press.

Csordas, Thomas J., 1990, «Embodiment as a Paradigm for Anthropology », Ethos 18 (1) : 5-42.

— 1994, Embodiment and Experience: The existential ground of culture and self. Cambridge, Cambridge University press.

Deleuze, Gilles, 1985, L'image-temps. Paris: Les Éditions de Minuit.

Jaibi, Fadhel, 1991, «Je fais du théâtre parce que je le déteste », Journal Arrai Elaam, 16 novembre.

Kerrou, Mohamed, 1998, «Politiques de l'Islam en Tunisie », dans Mondher Kilani (dir.), Islam et changement social. Lausanne, Payot: 81-102.

Legendre, Pierre, 1978, La Passion d'être un autre: Étude pour la danse. Paris, Seuil.

—., 1988, Le désir politique de Dieu : Études de montages de L'État et du Droit. Paris, Fayard.

— 1994, Dieu au miroir : Étude sur l'institution des images. Paris, Fayard.

— 1999, Miroir d'une nation: L'École Nationale d'Administration. Paris, Mille et une nuits.

—_, 2000, L'empire de la vérité : Introduction aux espaces dogmatiques industriels. Paris, Fayard. 
Léonardi, Cécile, 1999, «Y a-t-il du corps dans le corps qui danse?» dans Claude Feintz (dir.), Les imaginaires du corps, Tome II. Paris, L'Harmattan : 53-72.

Martin, Denis Constant, 2002, «Sur la piste des OPNI : Objets politiques non identifiés ». dans Denis Constant Martin (dir.), Sur la piste des OPNI. Paris, Karthala : 73-104.

Nora, Pierre, 1983, Les lieux de mémoire, Tome I. Paris, Gallimard.

Ricœur, Paul, 2003, La mémoire, l'histoire, l'oubli. Paris, Fayard. 